\section{POS1351 CAUSES OF HOSPITALIZATION IN BEHÇET SYNDROME}

Y. Ozguler ${ }^{1,2}$, G. Hatemi ${ }^{1,2}$, A. S. Pala ${ }^{3}$, S. N. Esatoglu ${ }^{1,2}$, S. Ugurlu, ${ }^{1,2}$, E. Seyahi ${ }^{1,2}$, M. Melikoglu $^{1,2}$, I. Fresko ${ }^{1,2}$, H. Ozdogan $^{1}$, S. Yurdakul $^{1,2}$, H. Yazici ${ }^{1,2}$, V. Hamuryudan ${ }^{1,2} .{ }^{1}$ Istanbul University-Cerrahpasa, Cerrahpasa Medical Faculty, Department of Internal Medicine, Division of Rheumatology, Istanbul, Turkey; ${ }^{2}$ Istanbul University-Cerrahpasa, Cerrahpasa Medical Faculty, Behcet Disease Center, Istanbul, Turkey; ${ }^{3}$ Prof. Dr. Cemil Taşcıŏglu City Hospital, Department of Internal Medicine, Istanbul, Turkey

Background: The causes of hospitalization may provide important information on the course of diseases and treatment-related adverse effects.

Objectives: We aimed to determine the causes and outcome of hospitalizations among patients with Behçet Syndrome (BS) in a dedicated center.

Methods: We surveyed hospitalization records in our clinic between January 2002 and December 2019 and identified those with a diagnosis of BS. The records of these patients were reviewed for demographic and clinical features, causes of hospitalization and outcome. We divided hospitalization causes into 2 as being BS related (organ involvement or deterioration) and non-BS related (treatment complication or others). Results: Three-hundred and thirty BS patients $(75 \%$ men, mean age $37.7 \pm 11.4$ SD years) were hospitalized for a total of 456 times during 18 years. The mean disease duration was $10.8 \pm 8.8$ SD years. Two-hundred and ninety-one (64\%) patients were using immunosuppressives (IS) with or without corticosteroids (CSs) and $72(16 \%)$ of them were under biologic treatment at the time of hospitalization. The mean duration of hospitalization was $12.7 \pm 10.7$ SD days. The reasons for hospitalization were directly related to BS in 259 patients $(57 \%)$ and non-related to BS in 191 (42\%). Six patients were hospitalized for both BS and non-BS related reasons at the same time. The most common reasons were vascular involvement $(n=169,64 \%)$ for BS related reasons and infections $(n=64$, $32 \%$ ) for non-BS related reasons (Table 1). Patients hospitalized for BS related causes were younger $(35.2 \pm 10.6$ vs $41.1 \pm 11.7, p<0.001)$, had short mean disease duration ( $8.5 \pm 7.5$ vs. $13.6 \pm 9.4$ years, $p<0.001)$, stayed shorter in the hospital (11.6 \pm 8.6 vs $14.0 \pm 12.9$ days $p=0.03)$ and had less frequent IS \pm CSs use $(59 \%$ vs $70 \%, \mathrm{p}=0.02)$ compared to those with non-BS related hospitalizations. There were no differences between the groups regarding gender distribution $(203 \mathrm{M} / 62 \mathrm{~F}$ vs. $143 \mathrm{M} / 54 \mathrm{~F}$ ) and use of biologic agents ( $15 \%$ vs $17 \%$ ). Three patients died during hospitalization. The reasons were malignancy, infection and right heart failure due to pulmonary artery thrombosis and pulmonary hypertension, respectively. Conclusion: Vascular involvement is the leading cause of hospitalization among BS patients, followed by infections. The predominance of men among

Table 1. Distributions of BS related and non-BS related reasons of hospitalizations

BS patients hospitalized with BS patients hospitalized with $B S$ related reasons non-BS related reasons ( $\mathrm{n}$ of pts=195, $\mathrm{n}$ of ( $\mathrm{n}$ of pts=170, $\mathrm{n}$ of

hospitalizations $=265)^{\star} \quad$ hospitalizations $\left.=197\right)^{\star}$

\begin{tabular}{|c|c|c|}
\hline $\begin{array}{l}\text { Causes of } \\
\text { hospitalizations } \\
\text { (per hospitalization) }\end{array}$ & $\begin{array}{l}\text { Vascular inv. }(n=169,64 \%) \\
\text { Pulmonary artery inv. } \\
\quad(n=64,24 \%) \\
\text { Deep vein thrombosis ( } n=39 \text {, } \\
\quad 15 \%) \\
\text { Budd-Chiari synd. }(n=24,9 \%) \\
\text { Vena cava inf. thrombosis } \\
\quad(n=19,7 \%) \\
\text { Peripheral artery inv. } \\
\quad(n=15,6 \%) \\
\text { Vena cava sup. thrombosis } \\
\quad(n=14,5 \%) \\
\text { Aorta inv. }(n=14,5 \%) \\
\text { Coronary artery inv. }(n=4,2 \%) \\
\text { Neurologic inv. }(n=50,19 \%) \\
\text { Parenchymal inv. }(n=37,14 \%) \\
\text { Dural sinus thrombosis } \\
\quad(n=13,5 \%)\end{array}$ & $\begin{array}{l}\text { Drug side effects other than } \\
\text { infections }(n=29,15 \%) \\
\text { Interferon }(n=10,5 \%) \\
\text { Azathioprine }(n=7,4 \%) \\
\text { Cyclosporine }(n=5,3 \%) \\
\text { Steroid }(n=3,2 \%) \\
\text { TNF antagonists }(n=3,2 \%) \\
\text { IVIG }(n=1,1 \%) \\
\text { Additional rheumatologic dis- } \\
\text { eases ( } n=17,9 \%) \\
\text { Renal disease }(n=16,8 \%) \\
\text { Cardiovascular dis. }(n=12,6 \%) \\
\text { Avascular necrosis }(n=4,2 \%) \\
\text { Malignancy }(n=11,6 \%) \\
\text { Others }(n=40,20 \%)\end{array}$ \\
\hline
\end{tabular}

*Some patients were hospitalized more than one times and for both BS related and non-BS related reasons at different time and had more than one type of BS related and/or non-BS related reasons. hospitalized patients underlines the relatively severe course of BS in men. The retrospective design and inclusion of patients who were hospitalized only in the rheumatology unit are limitations of this study.

Disclosure of Interests: None declared

DOI: 10.1136/annrheumdis-2021-eular.1987

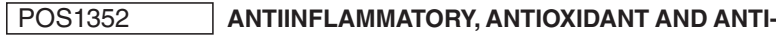 ATHEROSCLEROTIC EFFECTS OF A COMBINATION OF NATURAL SUPPLEMENTS ON PATIENTS WITH FMF RELATED AA AMYLOIDOSIS: A NON-RANDOMIZED 24 WEEKS OPEN LABEL INTERVENTIONAL STUDY}

M. Romano ${ }^{1}$, F. Garcia-Bournissen ${ }^{2}$, D. Piskin ${ }^{2}$, R. Cimaz ${ }^{3}$, M. Yilmaz ${ }^{4}$, E. Demirkaya ${ }^{1}$. ${ }^{1}$ Schulich School of Medicine \& Dentistry, University of Western Ontario, Paediatric Rheumatology, London, Canada; ${ }^{2}$ Western University, London, Ontario, Canada, Paediatrics, London, Canada; ${ }^{3}$ University of Milan, Department of Clinical Sciences and Community Health, Milan, Italy; ${ }^{4}$ Epigenetic Health Solutions, Unit of Nephrology, Ankara, Turkey

Background: Few studies have focused on Familial Mediterranean Fever (FMF)-related AA amyloidosis and cardiovascular disease event risk. Systemic inflammation stimulates the development and progression of atherosclerosis which is accelerated by vascular endothelial inflammation and enhanced oxidative stress. Excessive reactive oxygen species (ROS) generation has been reported in FMF, which correlated with attack severity. ROS may also be involved in amyloid formation, and in the pathogenesis of progressive renal injury.

Objectives: In this non-randomized, 24 weeks open label interventional study, we aimed to evaluate the effect of a combination of natural products on parameters related to inflammation, endothelial dysfunction and oxidative stress in a cohort of FMF patients with AA amyloidosis.

Methods: Morinda citrifolia (anti-atherosclerotic liquid- AAL), omega-3 (anti-inflammatory capsules- AIC) and extract with Alaskan blueberry (anti-oxidant liquid- $A O L$ ) were given to patients with FMF related $A A$ amyloidosis. We included patients with biopsy proven AA amyloidosis, older than 18 years who have nor mal estimated glomerular filtration rate (eGFR) and proteinuria [ $>3500 \mathrm{mg} /$ day] Flow-mediated dilatation (FMD), asymmetric dimethylarginine (ADMA), hs-CRP serum PTX3, Carotis intima media thickness (CIMT), malondialdehyde (MDA) $\mathrm{Cu} / \mathrm{Zn}$-superoxide dismutase (Cu/Zn-SOD), glutathione peroxidase (GSH-Px) levels were studied in baseline and after 24 weeks.

Results: 67 FMF-related amyloidosis [52 male (77.6\%); median recruitment age 36 years (range 21-66)] were enrolled. M694V mutation was the most common mutation found $(74 \%)$, with $50.7 \%$ of the patients in homozygosity. All patients were treated with colchicine, and most of them (83.6\%) has been on colchicine treatment at the time of enrollment. Serum ADMA, MDA, PTX3, hsCRP, cholesterol, and proteinuria were significantly decreased compared to baseline, while CuZn-SOD, GSH-Px and FMD levels were significantly increased following $A A L, A I C$ and $A O L$ therapies (Table 1). The change of the inflammatory markers PTX3, and hsCRP were negatively correlated with the change in FMD and positively correlated with the change of proteinuria, ADMA, MDA, cholesterol and CIMT.

Conclusion: 24 weeks of $\mathrm{AAL}, \mathrm{AIC}$ and $\mathrm{AOL}$ combined supplementation was significantly associated with reduction in serum inflammatory markers (PTX3 and hsCRP), improved endothelial functions (FMD, ADMA), and oxidative state (MDA). Our findings highlight the link among the three pathogenetic mechanisms including inflammation, endothelial function and oxidative status in progression of atherosclerosis and renal injury in patients with FMF related AA amyloidosis. Efficient control of these three mechanisms can have long term benefits from the cardiovascular and renal perspective of the patients with AA amyloidosis. REFERENCES:

[1] Romano M et al. Sci Rep, 2020

[2] Yilmaz M et al. Sci Rep, 2020

Table 1. Comparison of clinical and laboratory characteristics at the baseline and after 24 weeks of AAL, AIC and AOL supplementation

\begin{tabular}{lcccc}
\hline & Baseline & 24th week & Delta & \\
& & & & \\
& Mean $(\mathrm{SD})$ & Mean $(\mathrm{SD})$ & Mean $(\mathrm{SD})$ & $\mathrm{p}$ \\
\hline Total Cholesterol $(\mathrm{mg} / \mathrm{dl})$ & $221.2(60.3)$ & $155.8\left(35.4^{\star \star}\right)$ & $-65.3(55.5)$ & $<0.001$ \\
eGFR $(\mathrm{ml} / \mathrm{min} / 1.73 \mathrm{~m} 2)$ & $110.2(12.8)$ & $104.1\left(11.2^{\star *}\right)$ & $-6.1(11.9)$ & $<0.001$ \\
Malondialdehyde $(\mathrm{MDA})(\mathrm{nmol} / \mathrm{ml})$ & $4.2(1.8)$ & $1.8\left(0.5^{\star *}\right)$ & $-2.2(1.8)$ & $<0.001$ \\
CuZn-SOD $(\mathrm{U} / \mathrm{ml})$ & $431.5(154.7)$ & $538.1\left(146.4^{\star \star}\right)$ & $159.7(211.8)$ & $<0.001$ \\
GSH-Px $(\mathrm{U} / \mathrm{ml})$ & $47.8(13.2)$ & $74.1\left(20.3^{\star \star}\right)$ & $26.3(21.1)$ & $<0.001$ \\
ADMA $(\mu \mathrm{mol} / \mathrm{l})$ & $4.5(2.6)$ & $1.3\left(0.6^{* *}\right)$ & $-3.2(2.5)$ & $<0.001$ \\
FMD $(\%)$ & $5.0(0.7)$ & $6.4\left(0.8^{\star *}\right)$ & $1.3(0.9)$ & $<0.001$ \\
CIMT $(\mathrm{mm})$ & $0.9(0.2)$ & $0.7(0.1)$ & $-0.2(0.2)$ & $<0.001$ \\
Proteinuria $(\mathrm{mg} / 24 \mathrm{~h})$ & $6855.3(3116.9)$ & $4079.9(2359.6)$ & $-2775.3(2874.5)$ & $<0.001$ \\
hs-CRP $(\mathrm{mg} / \mathrm{l})$ & $25.5(4.4-48.0)$ & $3.0(1.0-9.1)^{\star}$ & $-20.8(11.2)$ & $<0.001$ \\
PTX3 $(\mathrm{ng} / \mathrm{ml})$ & $13.4(2.3-67.0)$ & $2.3(0.4-14.5)^{\star}$ & $-17.5(17.5)$ & $<0.001$ \\
\hline & & & & \\
\hline
\end{tabular}

